\title{
A review on hybridization modification of graphene and its polymer nanocomposites
}

\author{
ZHANG Chao \& LIU TianXi* \\ State Key Laboratory of Molecular Engineering of Polymers, Department of Macromolecular Science, Fudan University, Shanghai 200433, China
}

Received January 7, 2012; accepted March 4, 2012

Recently, graphene has attracted numerous interests from both fundamental and applied fields due to its excellent mechanical, thermal, electrical conductivity and other novel properties. This review gives an overview of recent progress on hybridization modifications of graphene with carbon nanomaterials. Some example applications of graphene-based nanohybrids in polymer composites, optical and conducting materials, high performance electrolyte materials and as well as other functional materials are summarized and discussed.

graphene, carbon nanomaterials, hybridization, architectures, polymer nanocomposites

Citation: Zhang C, Liu T X. A review on hybridization modification of graphene and its polymer nanocomposites. Chin Sci Bull, 2012, 57: 3010-3021, doi: 10.1007/s11434-012-5321-X

Graphene nanosheets (GNS), one of the emerging twodimensional (2D) carbon based nanomaterials in recent years [1-4], process a closely packed single-layered honeycomb lattice nanostructure composed of $\mathrm{sp}^{2}$ hybridized carbon atoms. GNS have a combination of excellent mechanical, electrical, and thermal properties which make them suit broad future applications such as in field-effect transistors [5-7], transparent electrodes [8,9], energy-storage materials [10-12], composites [13,14], chemical and biosensing [15,16], and many other areas [17]. In order to fully enlarge the graphene applications, large-scale, efficient, size-controlling and low-cost methods for graphene preparations are urgently needed and intensely studied. Up to now, graphene is mainly prepared by various methods including micromechanical cleavage [5], epitaxial growth [18,19], chemical vapor deposition (CVD) [20,21], and chemical oxidation-reduction methods [22-24]. Although the crystal structure of the GNS prepared by the traditional physical methods is relatively perfect and of integrity, the use of such methods requires more rigorous experimental conditions, and the yield of the graphene is rather low, which apparently do not suit to

*Corresponding author (email: txliu@ fudan.edu.cn) large-scale production of graphene and their further applications. Thus, taking the cost and processability of the graphene preparation into account, chemical oxidation-reduction method, generally derived from natural graphite, expandable graphite or derivatives of graphite (such as graphite oxide), has been providing a facile and processable platform for graphene applications.

Ideal structure of GNS is a complete two-dimensional crystal, and this inert surface structure also makes its process high chemical stability, large surface energy, weak interactions with other solvents. Thus the GNS are prone to agglomerate irreversibly due to strong van der Waals forces between GNS themselves. In order to obtain a stable dispersion of graphene, which not only improve the dispersion but also enhance its compatibility with the matrix, appropriate functionalization of graphene surface would be virtually important [25-33]. By far, a variety of functionalization methods including covalent and non-covalent modifications have been reported towards improving the solubility of GNS. For covalent modifications of graphene, the resultant products are rather stable. However, the complicated operations together with the harsh conditions of experiments greatly limit the bulk or mass production of graphene as 
well as their further applications. Compared with covalent grafting approaches, non-covalent modifications offer new ways to functionalize graphene without undergoing tedious chemical reactions. However, the stabilizing agents which can be utilized is limited. Simultaneously, by using such non-covalent modification methods, complete removal of the residual stabilizers is very difficult, which may greatly influence the properties (such as mechanical property and thermal stability) of the final products and thus their further applications. Therefore, finding suitable materials, which can simultaneously stabilize and form hybrids with graphene as well as be able to endow graphene with additional performance, has become new challenges toward graphene modifications and wide applications.

A hybrid material has been defined as a "material composed of an intimate mixture of inorganic components, organic components, or both types of components" [34]. New types of hybrid materials have been developed and used in both fundamental and applied fields. Recently, GNS decorated by metal and metal oxide nanoparticles is extensively studied and expected to be useful in catalysis, nanoelectronics, optics and nanobiotechnology. GNS-based hybrid materials with novel nanostructures and integrated properties have been intensively studied. Due to its excellent mechanical strength, high surface area, convenient and lowcost preparation method, GNS can be considered as one kind of potential nanoparticle carrier. Currently, the reports on nanoparticle-immobilized GNS hybrids have been increasing in exponential growth $[35,36]$. Among them, the combination of graphene with different dimensions of carbon-based materials showed a number of superior performances. In this review, we will summarize recent progress on the hybridization modification of graphene with carbon nanomaterials and their further applications in polymer composites.

\section{Structural properties of carbon nanomaterials}

Graphene has been viewed as a building block of all the other graphitic carbon allotropes with different dimensionality, as shown in Figure 1 [1]. For instance, graphite, threedimensional (3D) carbon allotrope, is made of GNS stacked on top of each other with a spacing of $0.33-0.34 \mathrm{~nm}$. Also, the zero-dimensional (OD) fullerenes can be considered to be made by wrapping a piece of GNS. The one-dimensional (1D) carbon allotropes, carbon nanotubes (CNTs) including single-walled carbon nanotubes (SWNTs) and multi-walled

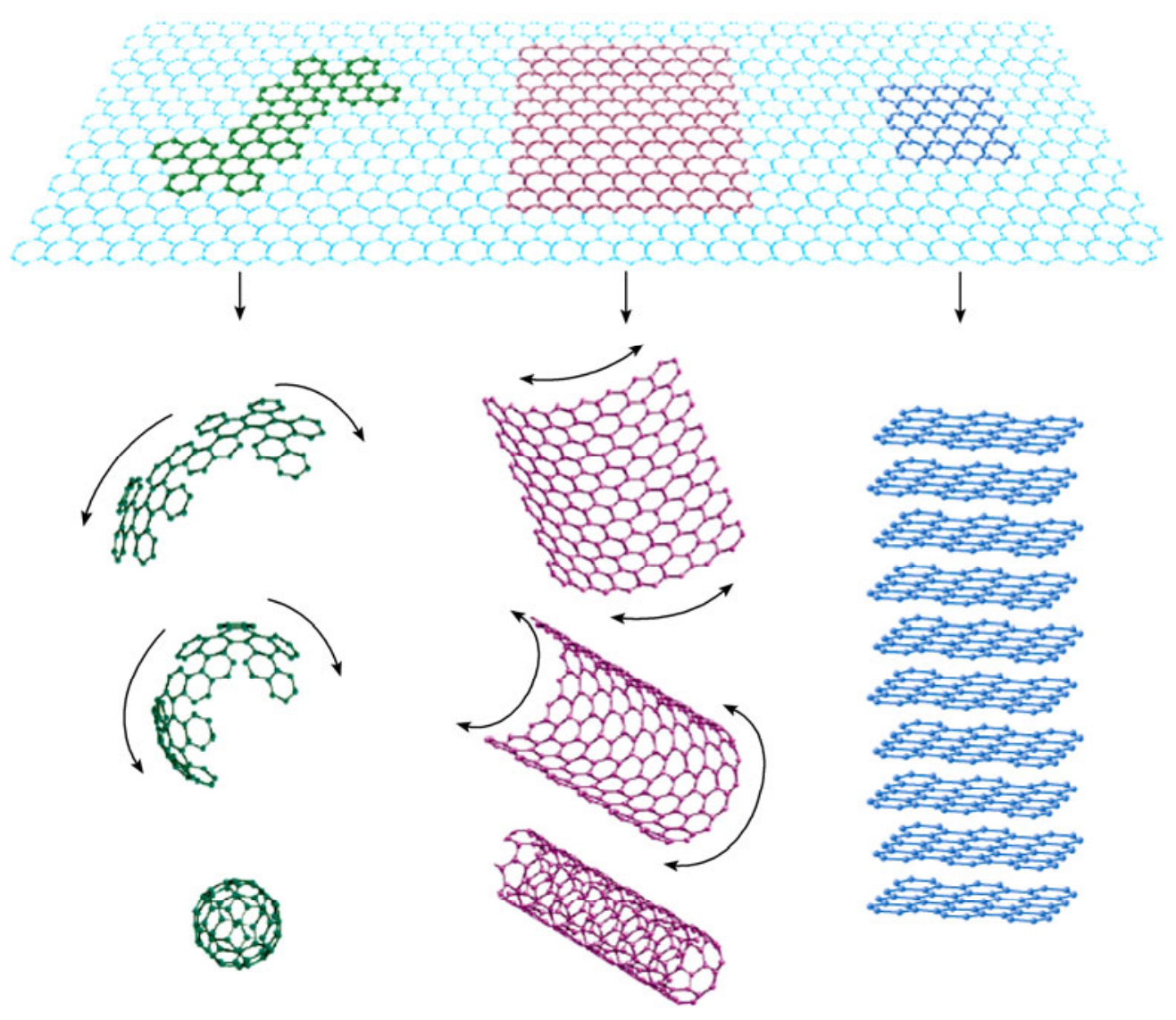

Figure 1 Graphene, the building block of all graphitic forms, can be wrapped to form the 0D buckyballs, rolled to form the 1D nanotubes, and stacked to form the 3D graphite. Reprinted with permission from [1], Copyright 2007, Nature Publishing Group. 
carbon nanotubes (MWNTs) can be made by rolling the GNS into single- or multi-walled tubular nanostructures. Table 1 presents the structural properties of several typical carbon nanomaterials including fullerene, SWNTs, MWNTs, GNS and graphite.

\section{Preparation strategies for the hybridization of graphene with carbon nanomaterials}

\subsection{Graphene-fullerene hybrids}

$\mathrm{C}_{60}$ and the higher fullerenes, building blocks to construct novel materials with outstanding properties, display remarkable chemical reactivity. Driven by the possibility of combining the outstanding properties of the fullerenes with those of other materials, various hybrid materials based on the fullerenes have generated intense attention. Novel electronic/optoelectronic or other properties for many potential applications of hybrid materials combining GNS and fullerenes were expected. Graphene-fullerene hybrids can be prepared through covalent bonding methods, as summarized below.

Chen and co-workers [37] reported a facile preparation of a graphene- $\mathrm{C}_{60}$ hybrid material via a simple coupling reaction between graphene oxide (GO) and pyrrolidine fullerene. The synthesis procedure for the $\mathrm{GO}-\mathrm{C}_{60}$ hybrid is shown in Figure 2. X-ray photoelectron spectroscopy demonstrates that one $\mathrm{C}_{60}$ molecule is covalently attached for every 130 carbon atoms of the $\mathrm{GO}^{-} \mathrm{C}_{60}$.

By using the same synthesis strategies, Chen and co-

Table 1 The structural properties of typical carbon nanomaterials including fullerene, SWNTs, MWNTs, GNS and graphite

\begin{tabular}{lccccc}
\hline & Fullerene & SWNTs & MWNTs & GNS & Graphite \\
\hline Dimensions & $0 \mathrm{D}$ & $1 \mathrm{D}$ & $1 \mathrm{D}$ & $2 \mathrm{D}$ & $3 \mathrm{D}$ \\
Length & $<1 \mathrm{~nm}$ & $\mu \mathrm{m}$ level & $\mu \mathrm{m}$ level & - & $\mu \mathrm{m}$ level \\
Thickness & $<1 \mathrm{~nm}$ & - & - & $<1 \mathrm{~nm}$ & $\mu \mathrm{m}$ level \\
Diameter & $<1 \mathrm{~nm}$ & $0.75-3 \mathrm{~nm}$ & $<100 \mathrm{~nm}$ & - & - \\
Density $\left(\mathrm{g} / \mathrm{cm}^{3}\right)$ & $\sim 1.7$ & $\sim 2.1$ & $\sim 2.1$ & $\sim 2.2$ & $\sim 2.62$ \\
\hline
\end{tabular}

workers [38] investigated the nonlinear optical properties of the graphene nanohybrid materials covalently functionalized with the fullerenes. Results show that covalently functionalizing graphene with the fullerene can enhance the nonlinear optical performance in the nanosecond regime, and the covalently linked graphene nanohybrids offer performance superior to that of the individual graphene and fullerene by combination of a nonlinear mechanism and the photoinduced electron or energy transfer between fullerene moiety and graphene (Figure 3).

\subsection{Graphene-CNT hybrids}

When dealing with the hybridization of typical 1D and 2D nanomaterials, the two components may have different topological architectures to form the nanohybrids. Therefore, according to the different architectures of the 2D GNS and the 1D CNTs, we reasonably classify the hybrids of the GNS and CNTs into three common types as illustrated in Figure 4. CNTs adsorbed horizontal to the GNS surface, CNTs adsorbed perpendicular to the GNS surface, and CNT wrapped with GNS.

In CNTs adsorbed horizontal to the GNS surface, GNS acted as a continuous sheet substrate for supporting the second component of CNTs. The majority of GNS-CNT hybrids were fabricated in this form. In CNTs adsorbed perpendicular to the GNS surface, possible reasons for the formation of this type of hybrid structure can be considered as a single interaction point between the GNS and CNT, namely, CNTs are connected with the GNS by the catalyst nanoparticles in CVD growth. In CNT wrapped with GNS, the formation of wrapping type depends on the interaction types between CNTs and GNS as well as the initial ratios between them. Table 2 summarizes the hybridization modification of GNS (or functionalized GNS) with pristine and functionalized CNTs in solvents, as reported in literature.

Liu and co-workers [40] found that the GO sheets consisting of multiple aromatic regions and hydrophilic oxygen groups can enhance the stability of pristine MWNTs in water. As shown in Figure 5, the MWNT-GO hybrids prepared by mild sonication and separation through simple sonicating

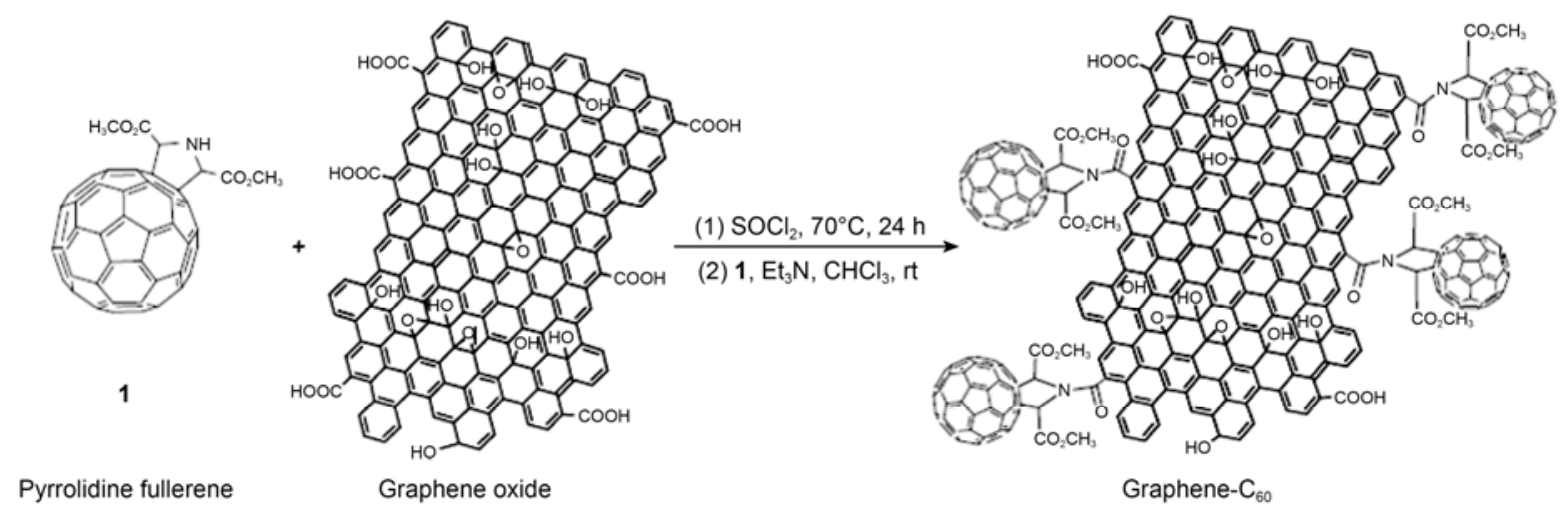

Figure 2 Synthesis procedure of the graphene- $\mathrm{C}_{60}$ hybrid material. Reprinted with permission from [37], Copyright 2008, Elsevier Science Ltd. 


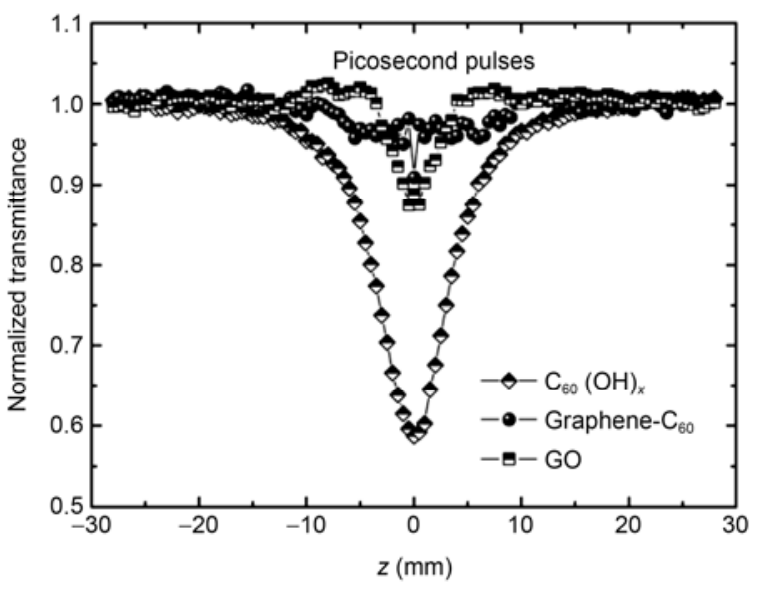

Figure 3 Open-aperture $Z$-scan curves of graphene- $\mathrm{C}_{60}$, pyrrolidine fullerene, and $\mathrm{GO}$ with picosecond pulses. Reprinted with permission from [38], Copyright 2009, American Chemical Society.

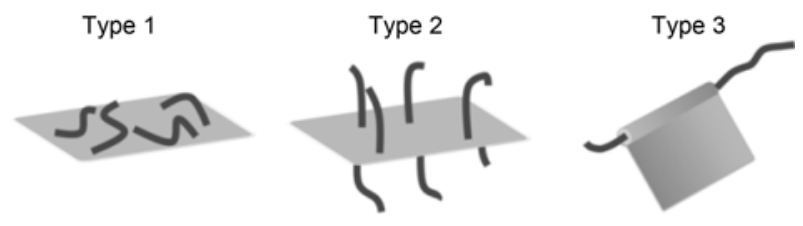

Figure 4 Three types of classifying the hybrids of graphene and CNTs.

and centrifugation, show good solubility in water. Also, the water-soluble and insoluble complexes can be obtained by changing the initial proportion of MWNTs to GO sheets (Figure 6), which are important for noncovalent approaches toward solubilizing CNTs and thus open a new way for GO applications in colloidal chemistry.

Chen and co-workers [42] prepared stable SWNT aqueous dispersion by using GO sheets as surfactant, and the SWNTs were completely wrapped by GO sheets to form a

Table 2 The hybridization modification of graphene with CNTs in solvents

\begin{tabular}{|c|c|c|c|c|c|c|}
\hline Graphene & $\mathrm{CNT}$ & Interaction & Architecture & Dispersion solvent & Weight ratio (G:CNT) & Ref. \\
\hline GO & SWNT & van der Waals interaction & Type 1 & Water & - & [39] \\
\hline GO & MWNT & $\pi-\pi$ stacking & Type 1 & Water & $>3$ & [40] \\
\hline GO & SWNT & - & - & Water & 0.5 & [41] \\
\hline GO & SWNT & $\pi-\pi$ stacking & Type 3 & Water & 2.5 & [42] \\
\hline GO & MWNT & $\pi-\pi$ stacking & Type 1 & Water & $>2$ & [43] \\
\hline GO & $\mathrm{NH}_{2}$-MWNT & Covalent bonding & Type 3 & Water & 40 & [44] \\
\hline GO salt & SWNT & van der Waals interaction & Type 1 & Water & $0.5-5$ & [45] \\
\hline GNS & SWNT & - & Type 1 & Hydrazine & 0.2 & [46] \\
\hline GNS & MWNT & CVD growth & Type 2 & - & - & [47] \\
\hline GNS & A-MWNT & $\pi-\pi$ stacking & Type 1 & Water & 1 & [48] \\
\hline GNS & A-MWNT & Ion-coordinated interaction & Type 1 & Water & $<0.25$ & [49] \\
\hline Graphene nanoplatelet (GNP) & SWNT & - & - & - & 3 & [50] \\
\hline $\begin{array}{l}\text { Chitosan (CS) grafted } \\
\text { GNS }\end{array}$ & MWNT & van der Waals interaction & Type 1 & Water & 1 & [51] \\
\hline
\end{tabular}
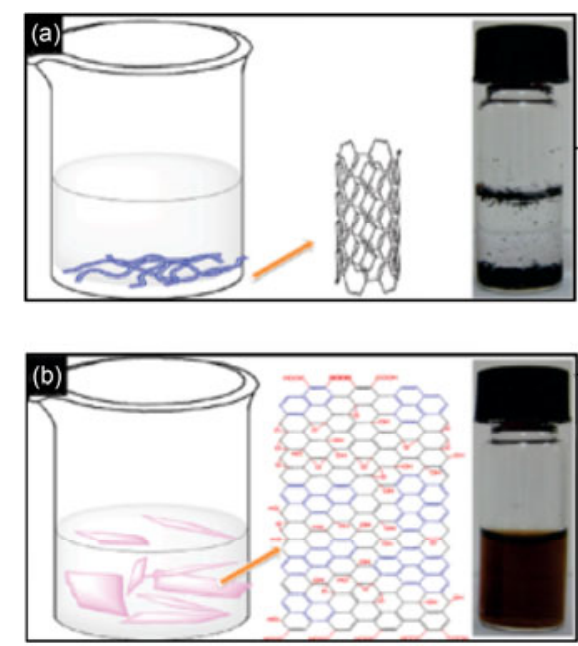

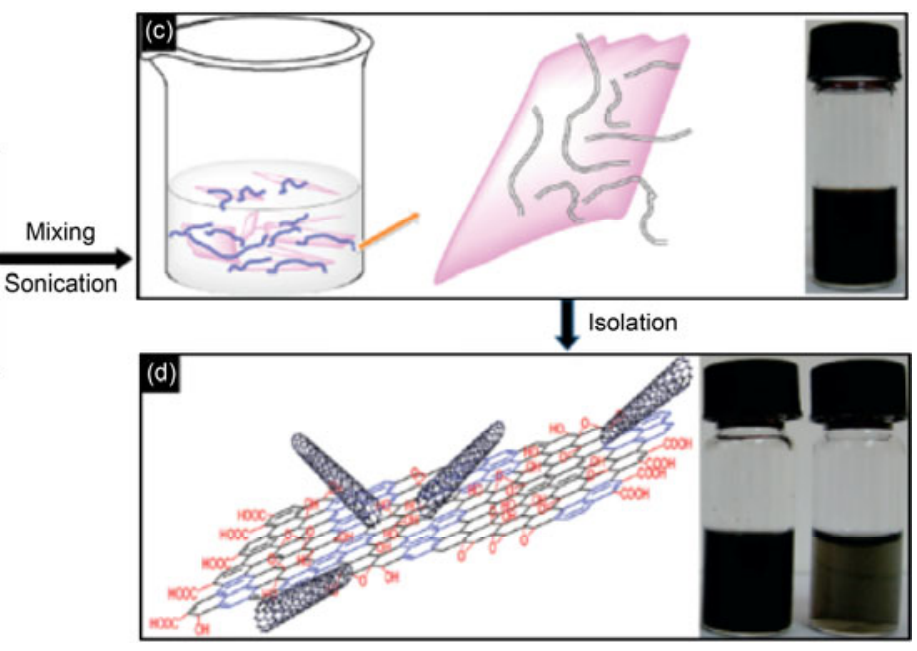

Figure 5 Schematic mechanism for formation of the GO-MWNT hybrids. Reprinted with permission from ref. [40], Copyright 2010, American Chemical Society. 

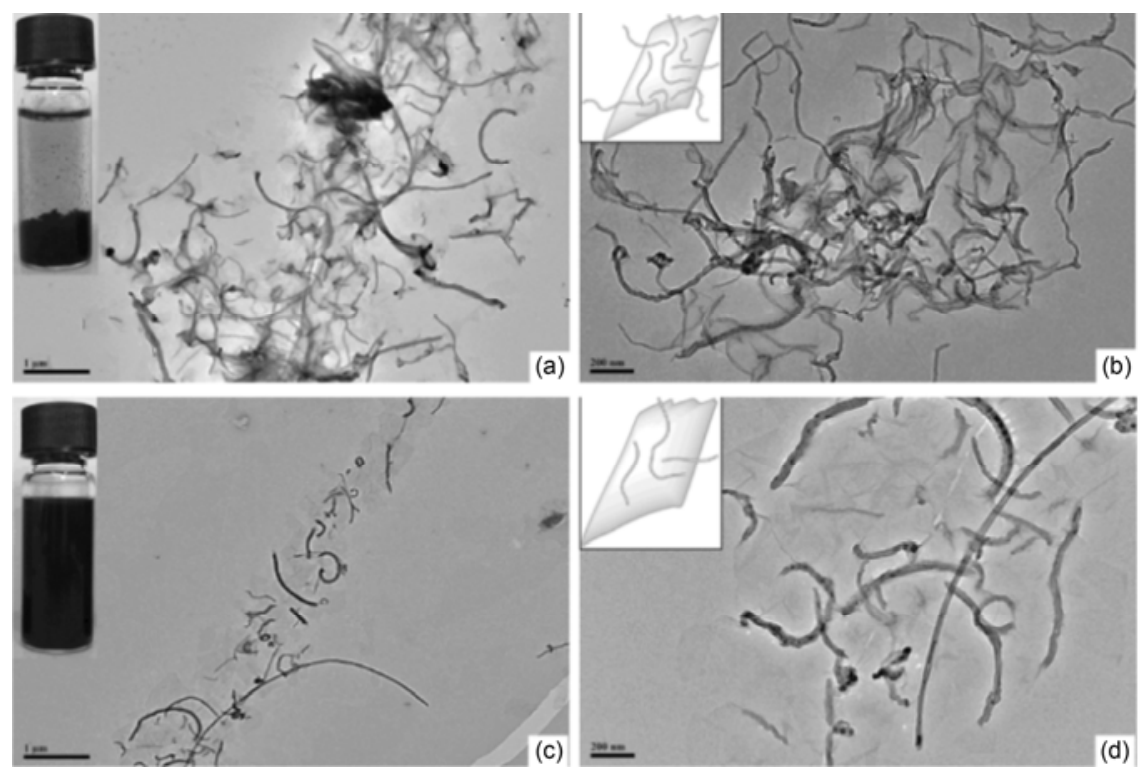

Figure 6 TEM images of interactions formed between MWNTs and GO sheets by changing the initial proportion of MWNTs to GO sheets with 2:1 at different magnifications ((a),(b)) and the initial proportion of MWNTs to GO sheets with 1:2 at different magnifications ((c),(d)). The inset in (b),(d) is the schematic description of the GO-MWNT hybrids. Reprinted with permission from [40], Copyright 2010, American Chemical Society.

core-shell structure, as viewed in Figure 7. This is the first time to experimentally demonstrate the spontaneous formation of SWNT/GO nanoscrolls and its potential applica- tions in optoelectronic devices and energy storage.

Scrolled GOs (SGO) with MWNT templates were prepared by Min and co-workers [44], and the synthesis
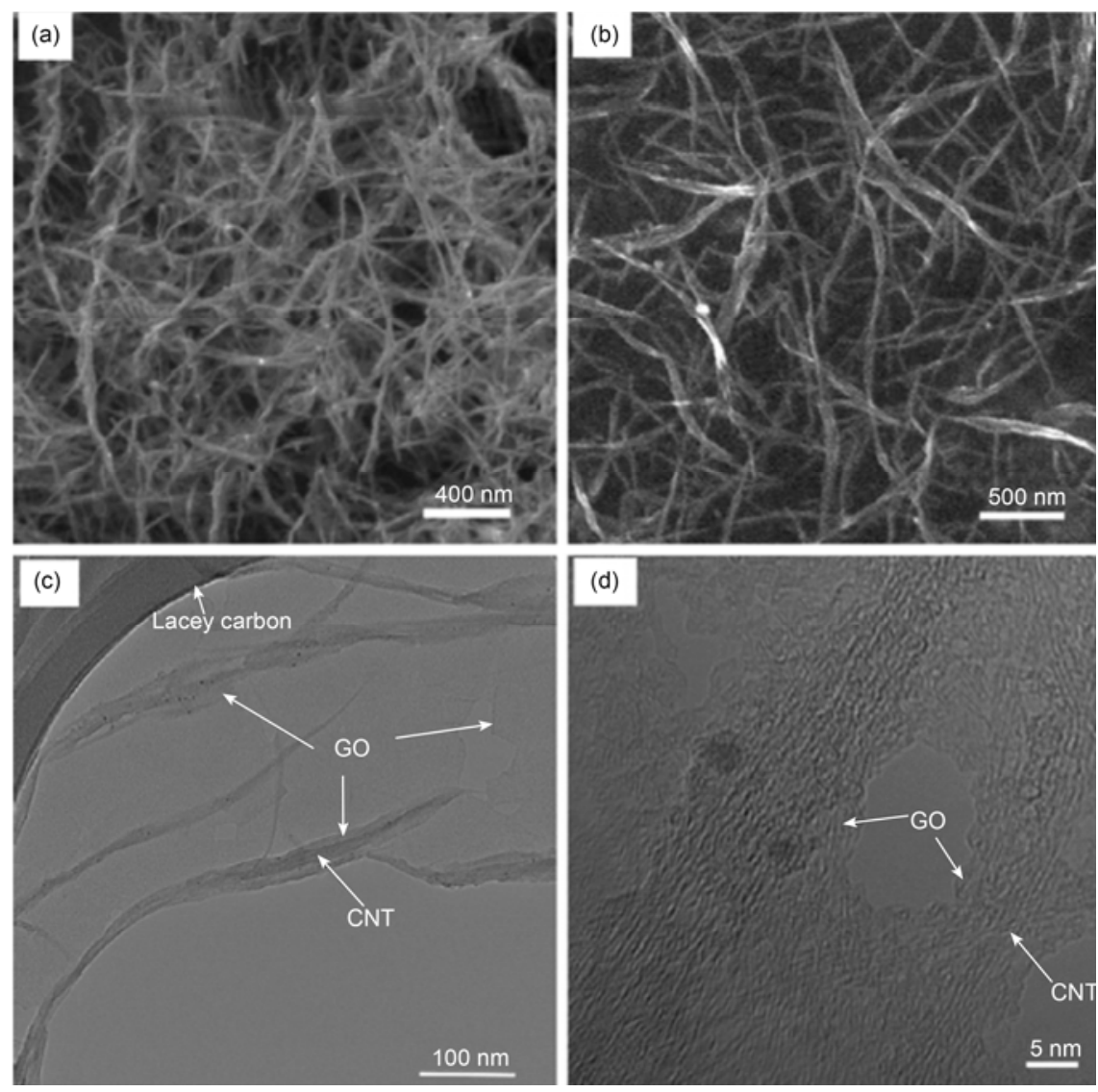

Figure 7 (a) SEM image of SWNTs; (b) SEM image of SWNT/GO; (c) TEM image of SWNT/GO; (d) high-resolution TEM image of SWNT/GO. Reprinted with permission from [42], Copyright 2011, Elsevier Science Ltd. 
strategies are shown in Figure 8. GO sheets were successfully made to adopt a scroll conformation around the surface of aminated MWNT in solution by covalent bond formation, which would allow large-scale production of SGO/MWNT hybrid materials as a good addition to existing MWNT- or GO-based hybrid materials.

Besides the hybridization of GO and pristine CNTs, the reduction of GO to GNS would be of great importance and also extensively studied. The formation of 3D GNS-CNT hybrids by the immobilization of metal catalysts onto single-layered GNS followed by in-situ CVD growth of CNTs has been reported. Zhao et al. [47] demonstrated the preparation of 3D CNT-pillared GO and GNS nanostructures with tunable length of the CNTs (Figure 9). Such nanostructures exhibited an excellent visible light photocatalytic performance in water. The cross-section views of the samples (Figure 10) clearly demonstrated a layered structure of the GO and GNS with CNTs as pillars between the GO and GNS platelets.

Liu and co-workers [48] prepared 3D GNS-MWNT hybrids by direct reduction of GO sheets in the presence of acid-treated MWNTs (A-MWNTs) shown in Figure 11. It is a simple and efficient method to fabricate novel water-dispersible GNS-MWNT hybrids due to $\pi-\pi$ stacking interactions between GNS and A-MWNTs. Direct evidence for the hybridization of the GNS with A-MWNTs can be obtained by TEM observation (Figure 12). One can clearly see the rolled edge of the GNS which gives a folded appearance, and in some regions the well-separated A-MWNTs were adsorbed on the GNS surface.
Xie et al. [49] reported that divalent metal ions $\left(\mathrm{M}^{2+}, \mathrm{M}=\right.$ $\mathrm{Cu}, \mathrm{Ca}$ or $\mathrm{Mg}$ ) are used to coordinate the oxidized MWNTs and GO by the oxygen-containing functional groups in a simple, solution-based route. Flexible and transparent conductive films of $\mathrm{M}^{2+}$-coordinated CNT/GO networks are produced by spin coating (Figure 13). This method provides a new route to transparent electrode materials with high flexibility and strength, which may find potential applications in nanoscale biosensors and catalyst-loaded reactors.

\subsection{Graphene-graphite nanoplatelet hybrids}

In some studies, GOs are not only effective dispersion agents for other carbon nanomaterials but also unconventional polymeric matrices for nanocomposites.

Sun and co-workers [52] used aqueous GOs to effectively disperse graphene nanoplatelet (GNP) in suspension for facile wet- processing into nanocomposites of GNP embedded in GO, as shown in Figure 14. The resulting lightweight and plastic-like nanocomposite materials remained mechanically flexible even at high loadings of GNP. The nanocomposites were found to be highly efficient in thermal transport, with the experimentally determined thermal diffusivity competitive to those typically observed only in wellknown thermally conductive metals such as aluminum and copper.

Yang and co-workers [53] fabricated macroscopic scale graphene-based membranes with controllable conductivity through a self-assembly approach at the liquid-air interface. In the assembling process, graphene oxide nanosheet (GON)

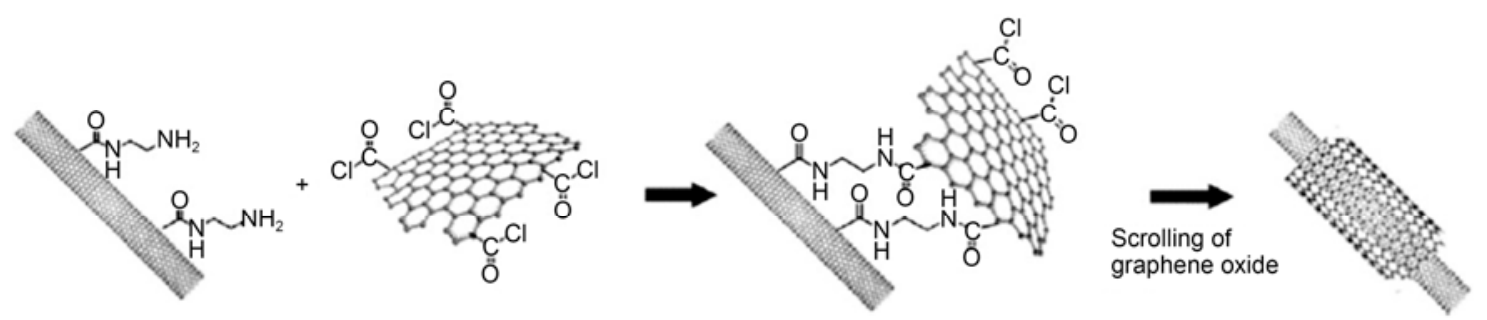

Figure 8 A scheme showing GO scroll formation around a MWNT template by covalent conjugation. Reprinted with permission from [44], Copyright 2010, Elsevier Science Ltd.

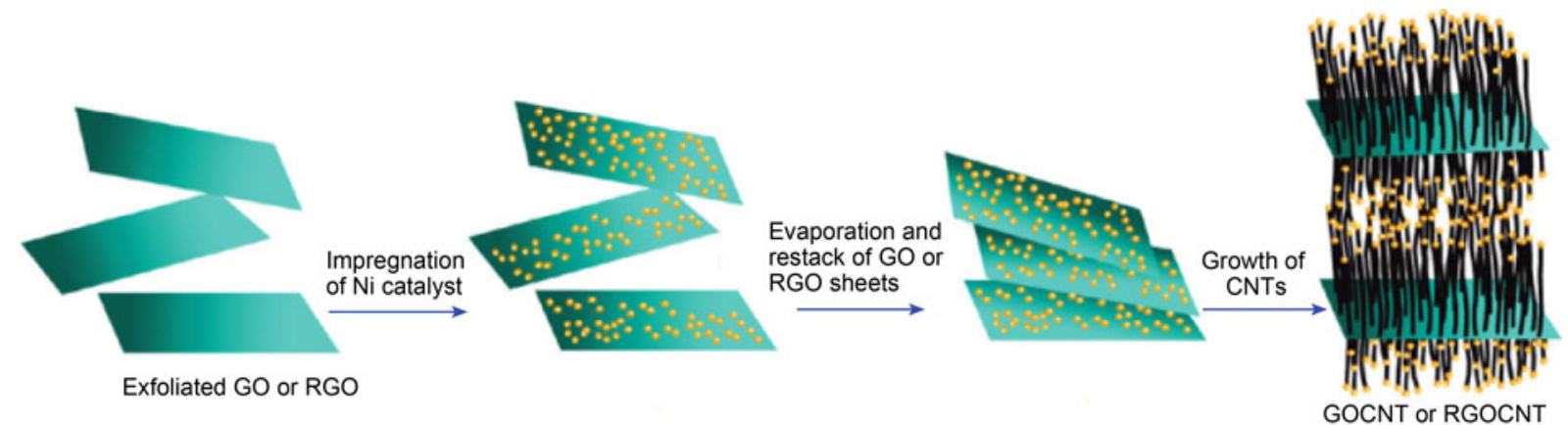

Figure 9 Experimental steps of pillaring GO and GNS platelets with MWNTs. Reprinted with permission from [47], Copyright 2010, American Chemical Society. 


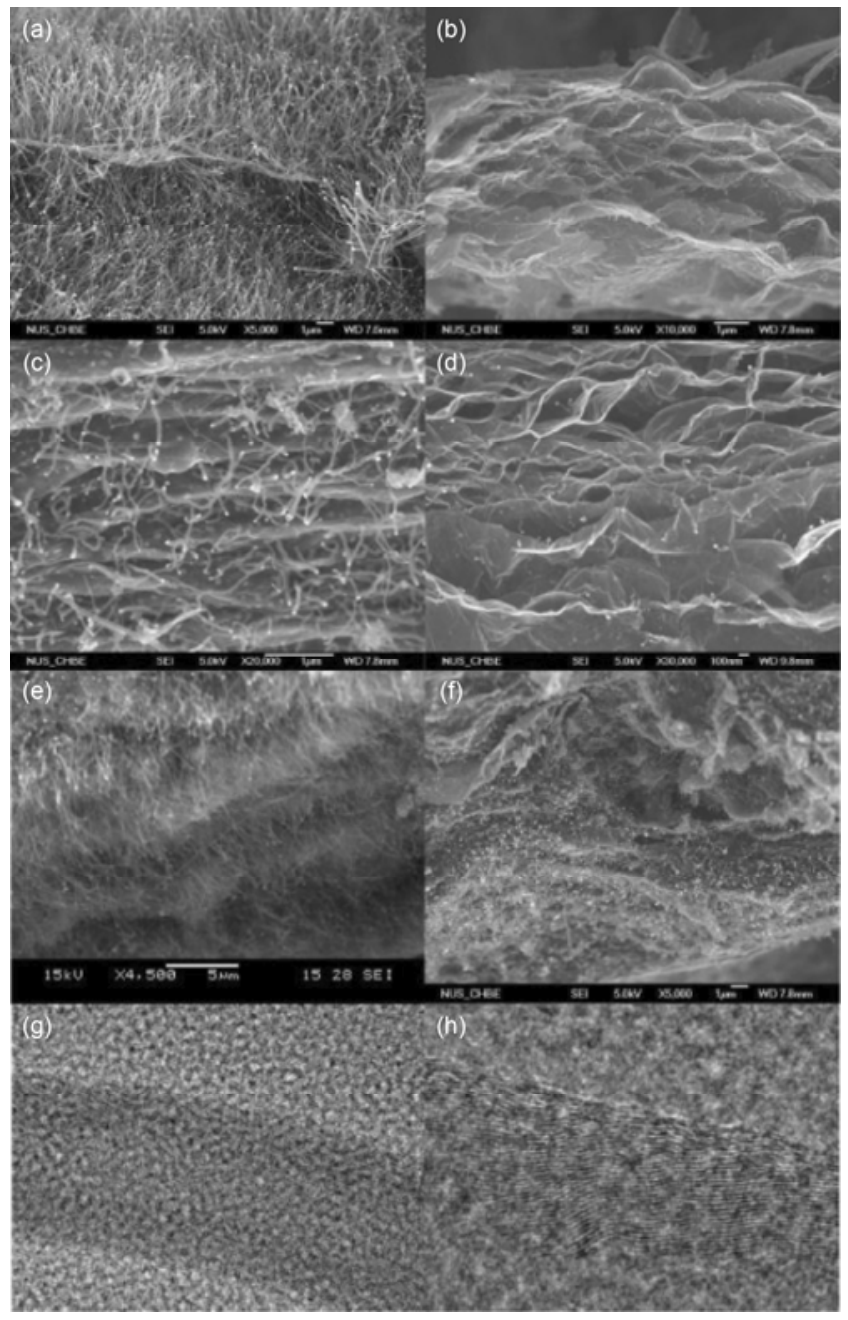

Figure 10 FESEM images of (a) GO:MWNT 30:17, (b) GO:MWNT 15:17, (c) GO:MWNT 30:9, (d) GO:MWNT 15:9, (e) GNS:MWNT 15:4, (f) GNS:MWNT 15:0.6 and TEM images of GO:MWNT 30:17 under different magnifications $((\mathrm{g})$ and $(\mathrm{h}))$. Reprinted with permission from [47], Copyright 2010, American Chemical Society. acts as the sticking component which binds the low-temperature exfoliated graphene nanosheets (LGN) together, and the interaction of GON and LGN promotes the assembly of layered structure and the formation of macroscopic membrane. Furthermore, the conductivity of membrane can be tuned by changing the GON fraction in the mixture suspension and the thickness of the membrane can be precisely controlled by the assembly period. According to the above results, the liquid-air interface self-assembly approach is an attractive and general strategy for the assembly of macroscopic graphene-based structure.

\section{Mechanical and electrical properties of gra- phene-based hybrid reinforced polymer nano- composites}

Theoretical and experimental results on individual GNS show that they process extremely high tensile modulus, tensile strength, good thermal and electrical conductivity. Combined with their low density, large aspect ratio, graphene are considered as outstanding candidates to substitute conventional nanofillers in the fabrication of high-performance and multi-functional polymer nanocomposites.

Haddon and co-workers [50] reported that a synergistic effect in the thermal conductivity enhancement of epoxy composites was achieved by combining 1D SWNT and 2D GNP fillers. They considered that the synergism originates from the bridging of planar nanoplatelets by the flexible SWNTs which lead to a decreased thermal interface resistance along the (2D-1D) hybrid filler network due to the extended area of the SWNT-GNP junctions. The SEM and TEM images of epoxy composite with a hybrid filler loading of GNP: SWNT of 3:1 (7.5 wt\% of GNPs and $2.5 \mathrm{wt} \%$ of SWNTs) are shown in Figure 15. The images show a
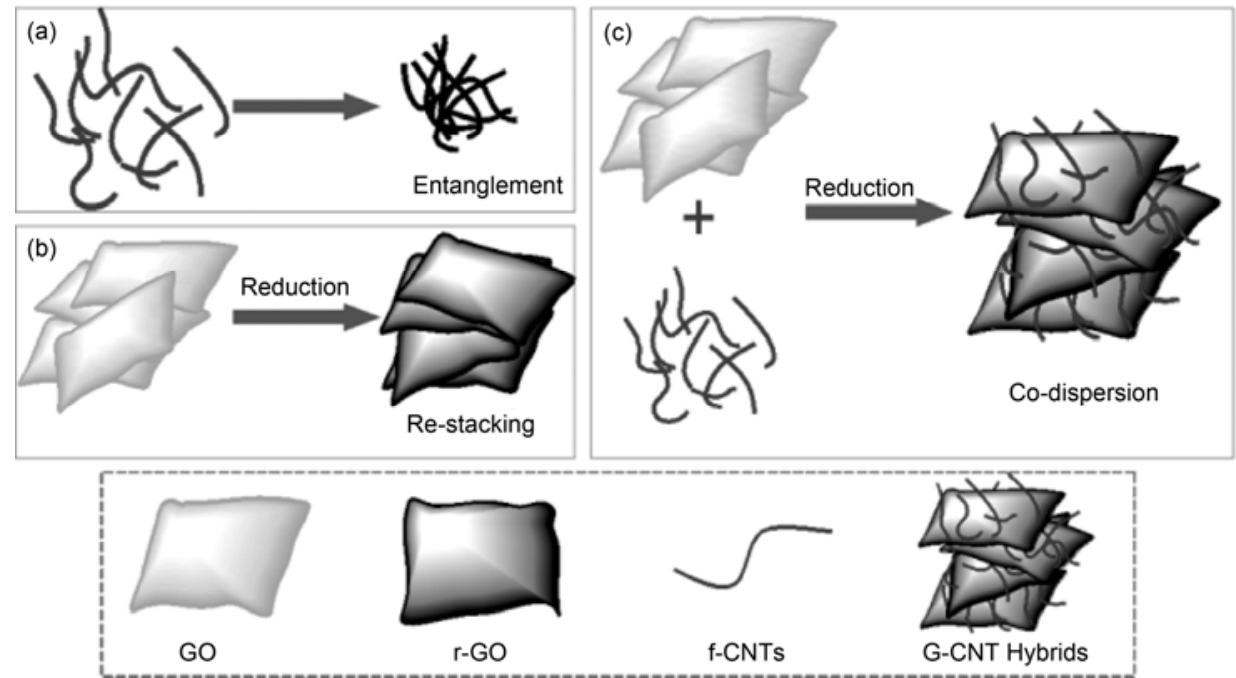

Figure 11 Schematic illustration for the co-dispersion mechanism of GNS with the aid of A-MWNTs [48]. Reproduced with permission of the Royal Society of Chemistry. 

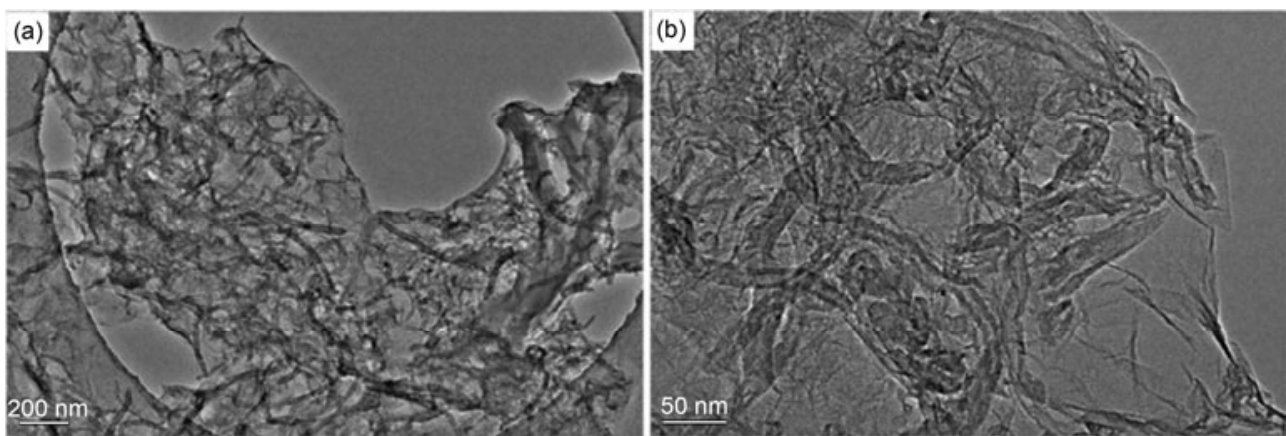

Figure 12 TEM images of GNS/A-MWNT (1:1) hybrids at (a) low and (b) high magnifications [48]. Reproduced with permission of the Royal Society of Chemistry.
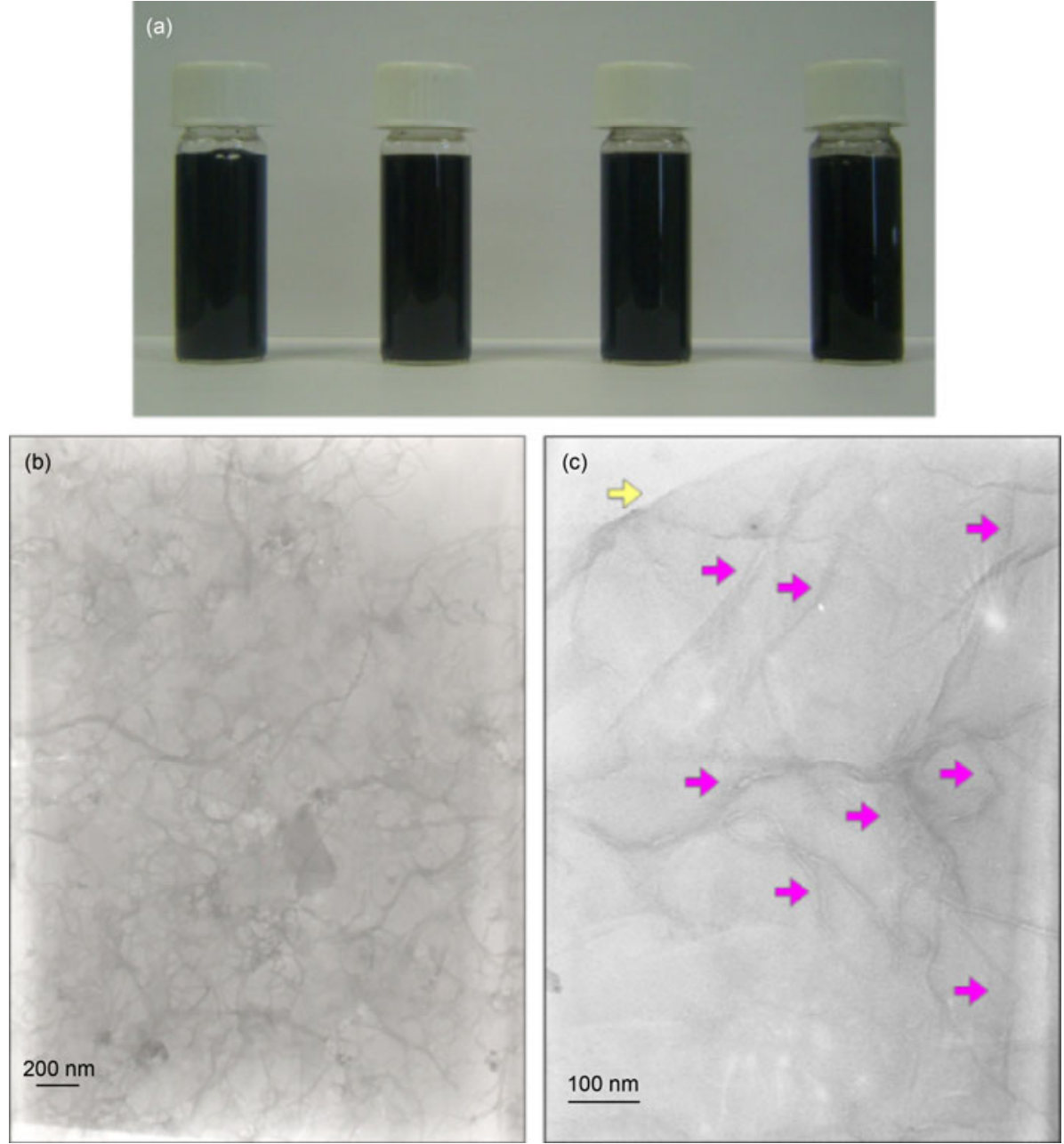

Figure 13 (Color online) (a) Photograph of $\mathrm{Cu}^{2+}$-coordinated MWNT/GO hybrid suspensions (75/25, 85/15, 90/10 and 100/0, from left to right), representative; (b) low- and (c) high-magnification TEM images of $\mathrm{Cu}^{2+}$-coordinated MWNT/CO hybrid films. In Figure 13(c), the yellow arrow points to the edge of a GO sheet, and the red arrows point to underlying MWNTs. Reprinted with permission from [49], Copyright 2011, Elsevier Science Ltd.

complex nanostructure with multiple SWNTs bridging adjacent GNPs. The hybrid filler thus provides the highest efficiency in the thermal conductivity enhancement of composites and can be utilized at low filler loading which is important for decreasing the viscosity and improving the processability of thermal interface materials.
Sun and co-workers [41] prepared SWNT, GO, graphene and their PVA hybrid fibers. Mixing SWNTs and GO improves dispersion of both in the polymer matrix and leads to the synergetic enhancement of the strength of the fibers. The conductivity of the fibers was also increased by mixing SWNTs and GO together at a ratio of $2: 1$. Contrary to GO 

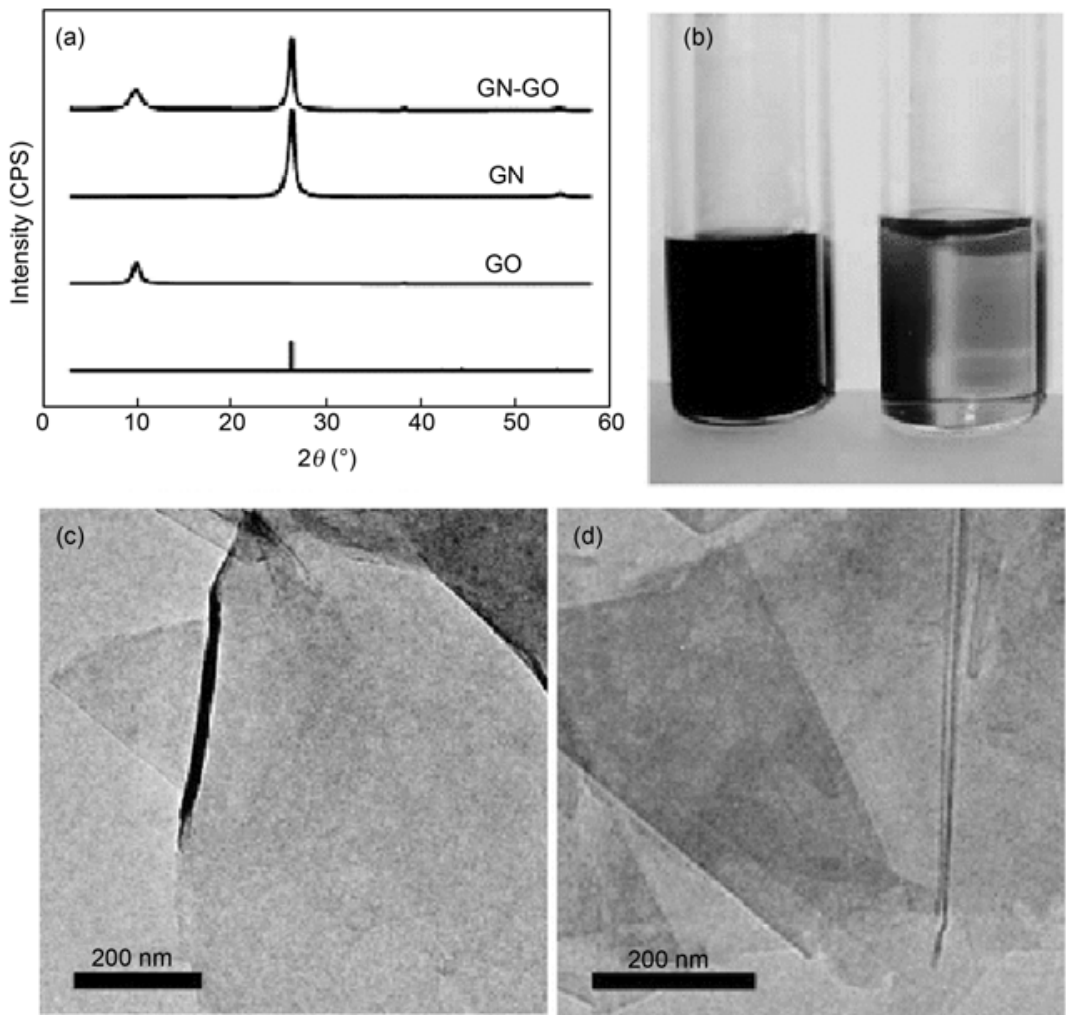

Figure 14 (a) X-ray diffraction patterns of the samples as marked are compared with that of bulk graphite. (b) Photos of aqueous solutions (left, concentrated; right, 1/10 diluted) of GOs from the Hummers method. Typical TEM images for specimens of GOs (c) and GNs (d). Reprinted with permission from [52], Copyright 2011, American Chemical Society.
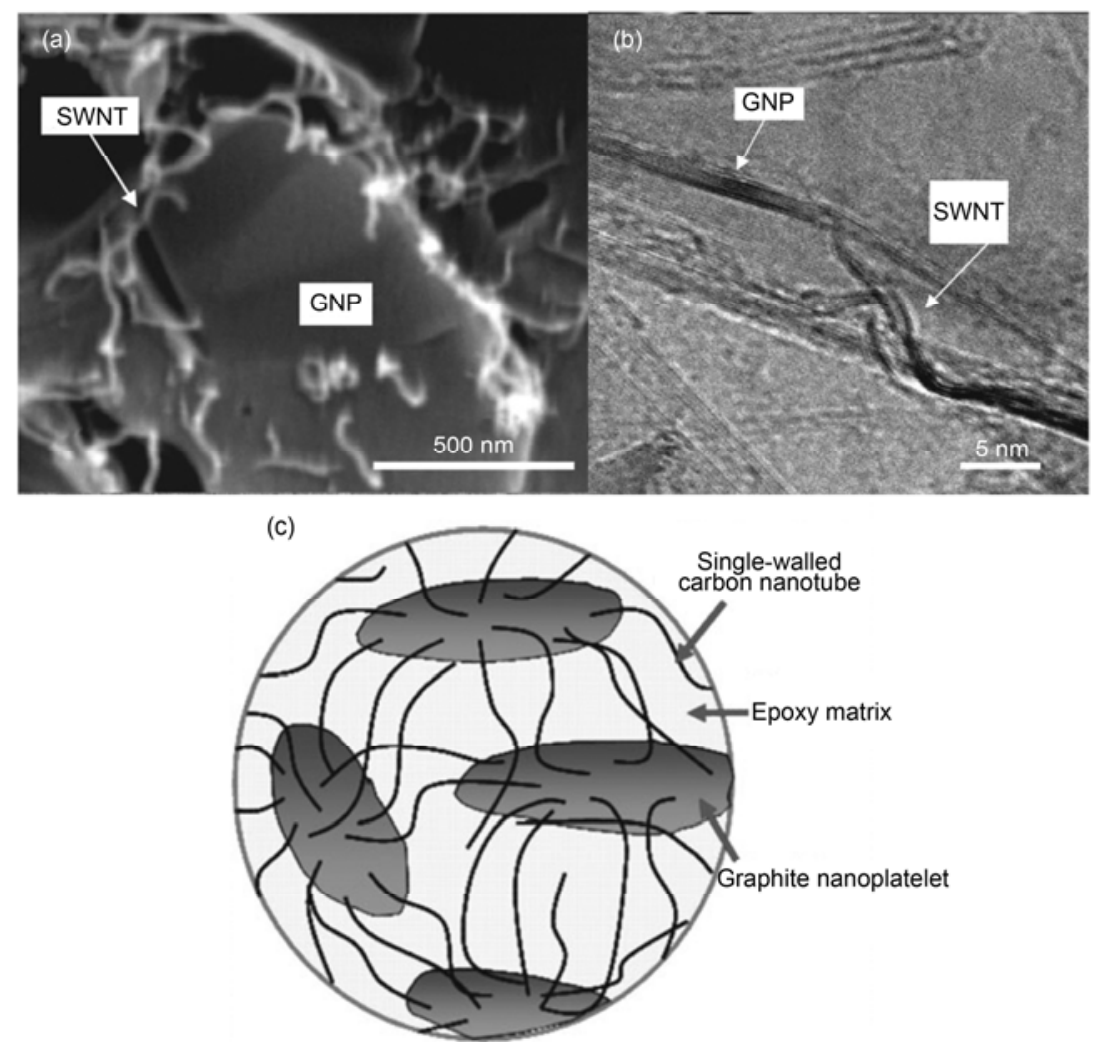

Figure 15 (a) SEM and (b) TEM images of the crosssection of GNP-SWNT hybrid filler/epoxy composite. (c) Schematic representation of GNP-SWNT network in polymer matrix. Reprinted with permission from [50], Copyright 2008, WILEY-VCH Verlag GmbH \& Co. KGaA, Weinheim. 
fibers, graphene fibers exhibit high toughness upon the removal of carboxyl and hydroxyl groups. Fibers with tunable toughness were designed by mixing SWNTs with graphene in different ratios. These tunable fibers have potential in a wide range of applications.

Liao and co-workers [43] demonstrated a simple and effective method to fabricate poly (vinyl alcohol) (PVA) composites containing MWNT-GO hybrids. By utilizing GO sheets as a dispersing agent, excellent dispersion of MWNTs in the PVA matrix has been achieved with the weight ratio of GO:MWNT about 2:1. Molecular dynamics simulations suggest that the interaction between GO and MWNT is strong and is thermodynamically favorable compared to the agglomeration of MWNTs. The PVA composite films containing GO dispersed CNTs show synergistic effects with superior mechanical properties than those of single GO or MWNT enhanced PVA composite films. The optimal mechanical properties of GO-MWNT/PVA were achieved with GO-CNTs content between $2 \mathrm{wt} \%-3 \mathrm{wt} \%$.

Liu et al. [48] prepared PVA composite films containing GNS-MWNT hybrids by a simple water casting method. Due to the homogeneous dispersion (see Figure 16) and synergistic interaction of the two kinds of nanofillers, the tensile strength and Young's modulus of the resulting PVA nanocomposite filled with only $0.6 \mathrm{wt} \% \mathrm{G}-\mathrm{CNT}$ hybrids are significantly improved by about $77 \%$ and $65 \%$, respectively. This work therefore provides a new way for the preparation of hybrid carbon nanomaterials with unique structure and excellent properties for fabricating high-performance polymer nanocomposites.

$\mathrm{Li}$ et al. [51] developed a green and facile method to prepare soluble GNS sheets by functionalizing them with chitosan (CS), which had a superior capability in dispersing and stabilizing MWNTs in acidic aqueous solutions via noncovalent interactions (Figure 17). The dispersed concentration of MWNTs could reach $0.41 \mathrm{mg} / \mathrm{mL}$ which is much higher than the values obtained in GO or sodium dodecyl sulfate (SDS) dispersed MWNT solutions under the same condition. Subsequently, the CS-GNS dispersed MWNTs were used to reinforce the CS in its nanocomposites and it was found that CS-GNS-MWNTs remarkably outperformed the other fillers for reinforcing and toughening of CS. With incorporation of $1 \mathrm{wt} \%$ CS-GNS-MWCNTs, the tensile modulus, strength and toughness of the nanocomposites could be increased by $49 \%, 114 \%$, and $193 \%$, respectively. The load transfer was attributed to three kinds of interfacial interactions: interaction between graphene sheets and nanotubes, covalent bond between graphene sheets and grafted CS, and hydrogen bonds between grafted CS and the CS matrix.

\section{Conclusion and prospective}

In summary, we have summarized the recent studies and progress on the hybridization modifications of graphene with carbon nanomaterials. With its unique structural and physical properties, graphene-based nanohybrids have attracted much attention in a variety of fields such as in polymer composite realm. To maximize the function of the graphene-based nanohybrids in polymer matrix, it requires the following: (1) it is a simple and processable method; (2) carbon nanoelements in the hybrids should have strong
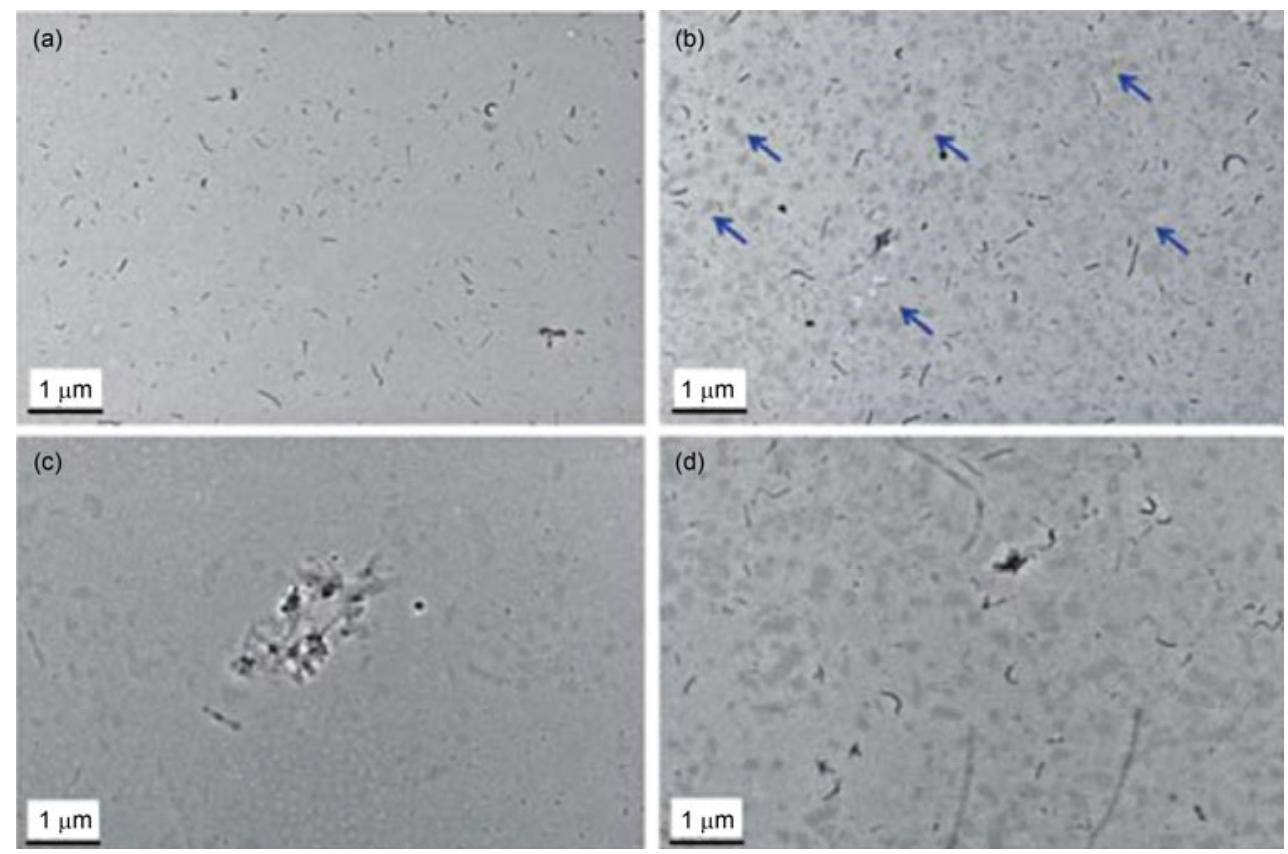

Figure 16 TEM images showing nanofiller dispersion from the ultrathin section of (a) PVA/MWNT, (b) PVA/(GNS-MWNT hybrids), (c) PVA/GNS, and (d) PVA/(GNS-MWNT mixture) composite films [48]. Reproduced by permission of the Royal Society of Chemistry. 

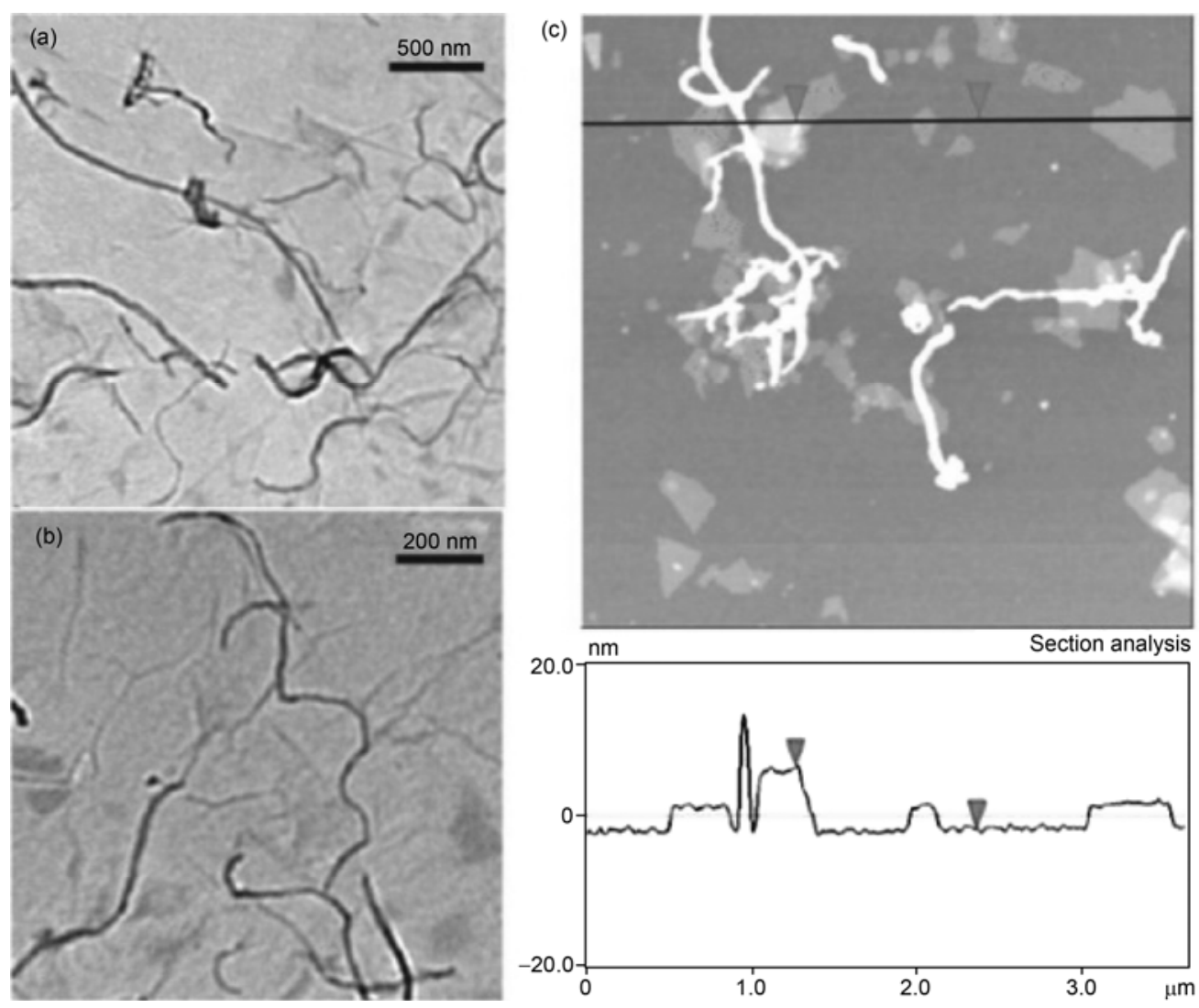

Figure 17 (a) and (b) TEM images of CS-g-GO/MWNTs under different magnifications; (c) AFM image with corresponding surface profile of CS-g-GO/MWNTs. Reprinted with permission from [51]. Copyright 2012, American Chemical Society.

interactions among them, which is beneficial to form efficient load transfer between the fillers themselves in the polymer matrix; (3) the nanostructured hybrids should show a much stronger interfacial interaction between the matrix and hybrid fillers than that with graphene alone. However, the existing investigations in the applications of graphenebased nanohybrids in polymer composites are still very limited and thus highly needed for this kind of novel materials together with computational studies stepping forward. Thus, future studies should focus on the following issues. First, low-cost and simple methods for the preparation of graphene-based hybrids, and their polymer composites are urgently needed. Second, besides the noncovalent hybridizations of graphene, covalent modifications between the graphene with other nanoelements can be further developed for polymer composites. Third, more potential applications of graphene hybrids reinforced polymer composites are pending to dig, such as in the fields of electro-magnetic shielding materials, biomaterials, and so on. With more and more investigations on this type of novel nanomaterials, we believe that graphene-based hybrids will bring us much surprise in the future.

This work was supported by the National Natural Science Foundation of China (51125011, 50873027) and "Shu Guang" Project (09SG02) of Shang- hai Municipal Education Commission and Shanghai Education Development Foundation.

1 Geim A K, Novoselov K S. The rise of graphene. Nat Mater, 2007, 6: 183-191

2 Li D, Kaner R B. Graphene-based materials. Science, 2008, 320: 1170-1171

3 Katsnelson M I. Graphene: Carbon in two dimensions. Mater Today, 2007, 10: 20-27

4 Rao C R, Biswas K, Subrahmanyam K S, et al. Graphene, the new nanocarbon. J Mater Chem, 2009, 19: 2457-2469

5 Novoselov K S, Geim A K, Morozov S V, et al. Electric field effect in atomically thin carbon films. Science, 2004, 306: 666-669

6 Cai W, Piner R D, Stadermann F J, et al. Large-area synthesis of high-quality and uniform graphene films on copper foils. Science, 2009, 324: 1312-1314

7 Li B, Cao X H, Ong H G, et al. All carbon electronic devices fabricated by directly grown single walled carbon nanotubes on reduced graphene oxide electrodes. Adv Mater, 2010, 22: 3058-3061

8 Zhao J P, Pei S P, Ren W C, et al. Preparation of large-area graphene oxide sheets for transparent conductive films. ACS Nano, 2010, 4: $5245-5252$

9 Kasry A, Kuroda M A, Martyna G J, et al. Chemical doping of large-area stacked graphene films for use as transparent, conducting electrodes. ACS Nano, 2010, 4: 3839-3844

10 Yang N, Zhai J, Wang D, et al. Two-dimensional graphene bridges enhanced photoinduced charge transport in dye-sensitized solar cells. ACS Nano, 2010, 4: 887-894

11 Arco L G, Zhang Y, Schlenker C W, et al. Continuous, highly flexible, and transparent graphene films by chemical vapor deposition for organic photovoltaics. ACS Nano, 2010, 4: 2865-2873 
12 Wang X, Zhi L J, Tsao N, et al. Transparent carbon films as electrodes in organic solar cells. Angew Chem Int Ed, 2008, 47: 29902992

13 Qi X Y, Pu K Y, Li H, et al. Amphiphilic graphene composites. Angew Chem Int Ed, 2010, 49: 9426-9429

14 Wang $\mathrm{H}$ L, Cui L F, Yang Y, et al. $\mathrm{Mn}_{3} \mathrm{O}_{4}$-graphene hybrid as a high-capacity anode materials for lithium ion batteries. J Am Chem Soc, 2010, 132: 13978-13980

15 Ang P K, Chen W, Wee A S, et al. Solution-gated epitaxial graphene as pH sensor. J Am Chem Soc, 2008, 130: 14392-14393

16 Dong X C, Shi Y M, Huang W, et al. Electrical detection of DNA hybridization with single-base specificity using transistors based on CVD-grown graphene sheets. Adv Mater, 2010, 22: 1649-1653

17 Hu W B, Peng C, Luo W J, et al. Graphene based antibacterial paper. ACS Nano, 2010, 4: 4317-4323

18 Srivastava S K, Shukla A K, Vankar V et al. Growth, structure and field emission characteristics of petal like carbon nano-structured thin films. Thin Solid Films, 2005, 492: 124-130

19 Zhu M Y, Wang J J, Outlaw R A, et al. Synthesis of carbon nanosheets and carbon nanotubes by radio frequency plasma enhanced chemical vapor deposition. Diamond Relat Mater, 2007, 16: 196-201

20 Wang J J, Zhu M Y, Outlaw R A, et al. Synthesis of carbon nanosheets by inductively coupled radio-frequency plasma enhanced chemical vapor deposition. Carbon, 2004, 42: 2867-2872

21 Berger C, Song Z, Li X B, et al. Electron confinement and coherence in patterned epitaxial graphene. Science, 2006, 312: 1191-1196

22 Stankovich S, Dikin D A, Piner R D, et al. Synthesis of graphene-based nanosheets via chemical reduction of exfoliated graphite oxide. Carbon, 2007, 45: 1558-1565

23 Dikin D A, Stankovich S, Zimney E J, et al. Preparation and Characterization of graphene oxide paper. Nature, 2007, 448: 457-460

24 Fan X, Peng W, Li Q, et al. Deoxygenation of exfoliated graphite oxide under alkaline conditions: A green route to graphene preparation. Adv Mater, 2008, 20: 1-4

25 Stankovich S, Piner R D, Chen X Q, et al. Stable aqueous dispersions of graphitic nanoplatelets via the reduction of exfoliated graphite oxide in the presence of poly (sodium 4-styrenesulfonate). J Mater Chem, 2006, 16: 155-158

26 Li X L, Wang X R, Zhang L, et al. Chemically derived, ultrasmooth graphene nanoribbon semiconductors. Science, 2008, 319: 12291232

27 Liang Y Y, WU D Q, Feng X L, et al. Dispersion of graphene sheets in organic solvent supported by ionic interactions. Adv Mater, 2009, 21: 1679-1683

28 Patil A J, Vickery J L, Scott T B, et al. Aqueous stabilization and self-assembly of graphene sheets into layered bio-nanocomposites using DNA. Adv Mater, 2009, 21: 3159-3164

$29 \mathrm{Xu} \mathrm{C}, \mathrm{Wu} \mathrm{X}, \mathrm{Zhu}$ J, et al. Synthesis of amphiphilic graphite oxide. Carbon, 2008, 46: 386-389

30 Wang S, Chia P J, Chua L L, et al. Band-like transport in surface-functionalized highly solution-processable graphene nanosheets. Adv Mater, 2008, 20: 3440-3446

31 Niyogi S, Bekyarova E, Itkis M E, et al. Solution properties of graphite and graphene. J Am Chem Soc, 2006, 128: 7720-7721

32 Lomeda J R, Doyle C D, Kosynkin D V, et al. Diazonium functionalization of surfactant-wrapped chemically converted graphene sheets. J Am Chem Soc, 2008, 130: 16201-16206

33 Georgakilas V, Bourlinos A B, Zboril R, et al. Organic functionalisation of graphenes. Chem Commun, 2010, 46: 1766-1768
34 Aleman J, Chadwick A V, He J, et al. Definitions of terms relating to the structure and processing of sols, gels, networks, and inorganicorganic hybrid materials. Pure Appl Chem, 2007, 79: 1801-1829

35 Guo S J, Dong S J. Graphene nanosheet: Synthesis, molecular engineering, thin film, hybrids, and energy and analytical applications. Chem Soc Rev, 2011, 40: 2644-2672

36 Singh V, Joung D, Zhai L, et al. Graphene based materials: Past, present and future. Prog Mater Sci, 2011, 56: 1178-1271

37 Zhang X, Huang Y, Wang Y, et al. Synthesis and characterization of a graphene-C60 hybrid material. Carbon, 2008, 47: 334-337

38 Liu Z B, Xu Y F, Zhang X Y, et al. Porphyrin and fullerene covalently functionalized graphene hybrid materials with large nonlinear optical properties. J Phys Chem B, 2009, 113: 9681-9686

39 Kim J, Cote L J, Kim F, et al. Graphene oxide sheets at interfaces. J Am Chem Soc, 2010, 132: 8180-8186

40 Zhang C, Ren L L, Wang X Y, et al. Graphene oxide-assisted dispersion of pristine multiwalled carbon nanotubes in aqueous media. $\mathbf{J}$ Phys Chem C, 2010, 114: 11435-11440

41 Wang R, Sun J, Gao L, et al. Fibrous nanocomposites of carbon nanotubes and graphene-oxide with synergetic mechanical and actuative performance. Chem Commun, 2011, 47: 8650-8652

42 Dong X, Xing G, Chan-Park M B, et al. The formation of a carbon nanotube-graphene oxide core-shell structure and its possible applications. Carbon, 2011, 49: 5071-5078

43 Li Y, Yang T, Yu T, et al. Synergistic effect of hybrid carbon nanotubegraphene oxide as a nanofiller in enhancing the mechanical properties of PVA composites. J Mater Chem, 2011, 21: 10844-10851

44 Kim Y K, Min D H. Preparation of scrolled graphene oxides with multi-walled carbon nanotube templates. Carbon, 2010, 48: 42834288

45 Tian L, Meziani M J, Lu F, et al. Graphene oxides for homogeneous dispersion of carbon nanotubes. ACS Appl Mater Interface, 2010, 2: 3217-3222

46 Tung V C, Chen L M, Allen M J, et al. Low-temperature solution processing of graphene-carbon nanotube hybrid materials for highperformance transparent conductors. Nano Lett, 2009, 9: 1949-1955

47 Zhang L L, Xiong Z, Zhao X S. Pillaring chemically exfoliated graphene oxide with carbon nanotubes for photocatalytic degradation of dyes under visible light irradiation. ACS Nano, 2010, 4: 7030-7036

48 Zhang C, Huang S, Tjiu W W, et al. Facile preparation of water-dispersible graphene sheets stabilized by acid-treated multi-walled carbon nanotubes and their poly(vinyl alcohol) composites. J Mater Chem, 2012, 22: 2427-2434

49 Liu Y T, Feng Q P, Xie X M, et al. The production of flexible and transparent conductive films of carbon nanotube/graphene networks coordinated by divalent metal ( $\mathrm{Cu}, \mathrm{Ca}$ or $\mathrm{Mg}$ ) ions. Carbon, 2011, 49: 3371-3375

$50 \mathrm{Yu}$ A, Ramesh P, Sun X, et al. Enhanced thermal conductivity in a hybrid graphite nanoplatelet-carbon nanotube filler for epoxy composites. Adv Mater, 2008, 20: 4740-4744

51 Pan Y, Bao H, Li L. Noncovalently functionalized multiwalled carbon nanotubes by chitosan-grafted reduced graphene oxide and their synergistic reinforcing effects in chitosan films. ACS Appl Mater Interfaces, 2012, 3: 4819-4830

52 Tian L, Anilkumar P, Cao L, et al. Graphene oxides dispersing and hosting graphene sheets for unique nanocomposite materials. ACS Nano, 2011, 5: 3052-3058

$53 \mathrm{Lv}$ W, Xia Z, Wu S, et al. Conductive graphene-based macroscopic membrane self-assembled at a liquid-air interface. J Mater Chem, 2011, 21: 3359-3364

Open Access This article is distributed under the terms of the Creative Commons Attribution License which permits any use, distribution, and reproduction in any medium, provided the original author(s) and source are credited. 